\title{
ENDS OF RIEMANNIAN MANIFOLDS WITH NONNEGATIVE RICCI CURVATURE OUTSIDE A COMPACT SET
}

\author{
MINGLIANG CAI
}

\begin{abstract}
We consider complete manifolds with Ricci curvature nonnegative outside a compact set and prove that the number of ends of such a manifold is finite and in particular, we give an explicit upper bound for the number.
\end{abstract}

\section{INTRODUCTION}

Toponogov [T] showed that in a complete manifold of nonnegative sectional curvature, a line splits off isometrically, i.e. any nonnegatively curved $M^{n}$ is isometric to a Riemannian product $N^{k} \times R^{n-k}$, where $N^{k}$ does not contain a line. Later, Cheeger and Gromoll [CG] generalized this to manifolds of nonnegative Ricci curvature, known as the Cheeger-Gromoll splitting theorem. As a consequence, such a manifold has at most two ends (see $\S 2$ for the definition of an end). In [A], Abresch studied manifolds with asymptotically nonnegative sectional curvature. He showed that the number of ends of such a manifold is finite and can be estimated from above explicitely. In this note, we consider manifolds with Ricci curvature being nonnegative outside a compact set and prove that the number of ends of such a manifold is finite and in particular, we give an explicit upper bound for the number. That is, we prove the following theorem.

Theorem. Let $\left(M^{n}, o\right)$ be a Riemannian manifold with base point $o$. If the Ricci curvature is nonnegative outside the geodesic ball $B(o, a)$ of radius $a$ and is bounded from below on $B(o, a)$ by $-(n-1) \Lambda^{2}$ (for $\left.\Lambda \geq 0\right)$, then there exists a universal bound on the number of ends, e.g.

the number of ends of $M^{n} \leq \frac{2 n}{n-1}(\Lambda a)^{-n} \exp \left(\frac{17(n-1)}{2} \Lambda a\right)$.

Received by the editors September 25, 1990 and, in revised form, October 9, 1990.

1980 Mathematics Subject Classification (1985 Revision). Primary 53C20. 
We learned that P. Li and L. F. Tam proved a similar theorem as an application of the theory of harmonic functions on a complete manifold. Our approach here is more geometrical. A previous version of the Theorem, under the additional condition of a lower bound on the sectional curvature, was proved by $\mathrm{Z}$. Liu. After reading a preliminary version of our paper, $Z$. Liu informed us that he could also modify his proof, using ideas from this paper, to prove the same theorem as above (see [LT, L]).

I would like to thank Professor DaGang Yang for bringing this problem to my attention and for some discussions I had with him. I would like to thank my advisor Professor Wolfgang Ziller for encouragement and guidance. I would also like to thank Tobias Colding for his interest in this work and for sharing his time and ideas with me in organizing this paper.

\section{IDEA OF THE PROOF OF THE THEOREM}

In what follows, we always let $M^{n}$ be a manifold as in the Theorem.

There are various (but equivalent) definitions of an end of a manifold (cf. [A]), for the sake of our argument, we use the following definition.

Definition 2.1. Two rays $\gamma_{1}$ and $\gamma_{2}$ starting at the base point $o$ are called cofinal if for any $r>0$ and any $t \geq r, \gamma_{1}(t)$ and $\gamma_{2}(t)$ lie in the same component of $M-B(o, r)$. An equivalence class of cofinal rays is called an end of $M$. We will use $[\gamma]$ to denote the class of the ray $\gamma$.

The following proposition is a key to the proof of the theorem.

Proposition 2.2. Let $M^{n}$ be as in the theorem, $\left[\gamma_{1}\right]$ and $\left[\gamma_{2}\right]$ be two different ends of $M^{n}$, then $d\left(\gamma_{1}(4 a), \gamma_{2}(4 a)\right)>2 a$.

Proposition 2.2 will be proved in $\S 3$. Assuming it, we now give a proof of the theorem.

Proof of the theorem. Let $k$ be an integer and $\gamma_{1}, \ldots, \gamma_{k}$ be rays from the base point $o$ going to $k$ different ends. We need to bound $k$ from above. Consider the sphere $S(o, 4 a)$ of radius $4 a$. Let $\left\{p_{j}\right\}$ be a maximal set of points on $S(o, 4 a)$ such that the balls $B\left(p_{j}, \frac{1}{2} a\right)$ are disjoint. Clearly, the balls $B\left(p_{j}, a\right)$ cover $S(o, 4 a)$, and since the set $\left\{\gamma_{i}(4 a), i=1, \ldots, k\right\}$ is contained in $S(o, 4 a)$, each $\gamma_{i}(4 a)$ is contained in some $B\left(p_{j}, a\right)$. But each ball $B\left(p_{j}, a\right)$ contains at most one $\gamma_{i}(4 a)$ by the Proposition 2.2, 
and hence the number of balls is not less than $k$. Thus it suffices to bound the number of balls $B\left(p_{j}, \frac{1}{2} a\right)$.

Notice that

$$
B\left(p_{j}, \frac{1}{2} a\right) \subset B\left(o, \frac{9}{2} a\right) \subset B\left(p_{j}, \frac{17}{2} a\right) .
$$

It follows from the Bishop-Gromov volume comparison theorem that

$$
\operatorname{vol} B\left(p_{j}, \frac{17}{2} a\right) \leq \frac{\int_{0}^{17 a / 2} \sinh ^{n-1} \Lambda t d t}{\int_{0}^{1 a / 2} \sinh ^{n-1} \Lambda t d t} \operatorname{vol} B\left(p_{j}, \frac{1}{2} a\right) .
$$

Therefore, the number of balls $B\left(p_{j}, \frac{1}{2} a\right)$ is no more than

$$
\frac{\int_{0}^{\frac{13}{2} a} \sinh ^{n-1} \Lambda t d t}{\int_{0}^{\frac{1}{2} a} \sinh ^{n-1} \Lambda t d t} .
$$

Since

$$
\frac{\int_{0}^{17 a / 2} \sinh ^{n-1} \Lambda t d t}{\int_{0}^{1 a / 2} \sinh ^{n-1} \Lambda t d t} \leq \frac{2 n}{n-1} \frac{e^{\frac{17(n-1)}{2} \Lambda a}}{(\Lambda a)^{n}},
$$

the theorem follows.

Remark 2.3. The bound for the number of ends given here is far from being sharp. An improved bound can be obtained from a more general volume comparison theorem which we can state as follows (for definitions involved, one is refered to [AG]):

A volume comparison theorem. Let $M^{n}$ be an asymptotically nonnegatively Ricci curved manifold. Then for any $p \in M^{n}$ and for every $0 \leq r \leq R$,

$$
\frac{\operatorname{vol} B(p, R)}{\operatorname{vol} B(p, r)} \leq w_{n}\left(\frac{R}{r}\right)^{n}
$$

where $w_{n}=(1+2 u(0) d(o, p))^{n-1} 2^{2 n} \exp \left(6(n-1) C_{1}\right)$.

Moreover, if $0 \leq r \leq R \leq d(o, p)$ or $2 d(o, p) \leq r \leq R, w_{n}$ can be chosen as $2^{2 n} \exp \left(6(n-1) C_{1}\right)$ (see [AG] for the definitions of $u(0)$ and $\left.C_{1}\right)$.

The proof of this theorem will appear elsewhere.

Proof of Proposition 2.2. Let $M$ be a manifold as in the theorem. 
For each ray $\gamma$, there is an associated function called the Busemann function, which is defined as follows:

$$
b_{\gamma}(x)=\lim _{t \rightarrow \infty}(t-d(x, \gamma(t))) \text {. }
$$

For any given point $p$, let $\alpha_{t}$ be a minimizing geodesic from $p$ to $\gamma(t)$. As $t \rightarrow \infty, \alpha_{t}$ has a convergent subsequence which converges to a ray at $p$. Such a ray is called an asymptotic ray to $\gamma$ at $p$.

Let $\gamma$ be a line. We define $\gamma^{+}:[0, \infty] \rightarrow M$ by $\gamma^{+}(t)=\gamma(t)$ and $\gamma^{-}:[0, \infty] \rightarrow M$ by $\gamma^{-}(t)=\gamma(-t)$.

Let $b_{\gamma}^{+}\left(b_{\gamma}^{-}\right.$, resp. $)$be the associated Busemann function of $\gamma^{+}\left(\gamma^{-}\right.$, resp $)$.

In [EH], J. Eschenburg and E. Heintze showed, under the assumption that the Ricci curvature is nonnegative everywhere, that $b_{\gamma}^{ \pm}$are smooth harmonic functions with Hess $b_{\gamma}^{ \pm}=0$ and $b_{\gamma}^{+}+$ $b_{\gamma}^{-}=0$. Applying their arguments locally, we can show the following lemma.

Lemma 3.1. Let $N$ be the $\delta$-tubular neighborhood of $\gamma$. Suppose that from every point $p$ in $N$, there is an asymptotic ray to $\gamma^{+}$and an asymptotic ray to $\gamma^{-}$such that the Ricci curvature is nonnegative on both asymptotic rays. Then through every point in $N$, there is a line $\alpha$ which, when parametrized properly, satisfies

$$
b_{\gamma}^{+}\left(\alpha^{+}(t)\right)=t \text { and } b_{\gamma}^{-}\left(\alpha^{-}(t)\right)=t \text {. }
$$

Proof. Let $p$ be any point in $N$. Applying arguments as in the proof of Lemma 3 in [EH], we find that at $p, b_{\gamma}^{+}+b_{\gamma}^{-}=0$, and $b_{\gamma}^{ \pm}$are $C^{1}$ smooth with $\left\|\operatorname{grad} b_{\gamma}^{ \pm}\right\|=1$. Hence the asymptotes to $\gamma^{ \pm}$are uniquely determined at $p$ and fit together to a line, say, $\gamma_{p}$. Arguments as in the proof of Lemma 2 together with the concluding remarks in [EH] imply that $b_{\gamma}^{+}\left(b_{\gamma}^{-}\right.$,resp.) is actually $C^{\infty}$ smooth with Hess $b_{\gamma}^{ \pm}=0$ on $\gamma_{p}$. Thus the restriction of $b_{\gamma}^{ \pm}$to $\gamma_{p}$ must be a linear function with derivative 1 . After a reparametrization of $\gamma_{p}$, Lemma 3.1 then follows.

Remark 3.2. The same argument as in [EH] of course also implies a local splitting for the metric in $N$, under the assumptions of Lemma 3.1 .

Lemma 3.3. $M^{n}$ cannot admit a line $\gamma$ with the following property:

$$
d(\gamma(t), B(o, a)) \geq|t|+2 a \text { for all } t .
$$


Proof. Suppose there were such a line $\gamma$. Consider the $a$-tubular neighborhood of $\gamma$. We claim that from any point $p$ in this neighborhood, all its asymptotic rays to $\gamma^{+}$(or $\gamma^{-}$) are away from $B(o, a)$, in particular, the Ricci curvature is nonnegative on such a ray. In fact, let $s$ be such that $d(p, \gamma(s))<a$, then,

$$
\begin{aligned}
d\left(p, \gamma^{ \pm}(t)\right) & \leq d(p, \gamma(s))+d\left(\gamma(s), \gamma^{ \pm}(t)\right) \\
& =d(p, \gamma(s))+d(\gamma(s), \gamma( \pm t)) \\
& \leq a+|s|+t
\end{aligned}
$$

but any curve from $p$ to $\gamma^{ \pm}(t)$ passing through $B(o, a)$ has length

$$
\begin{aligned}
l & \geq d(p, B(o, a))+d\left(\gamma^{ \pm}(t), B(o, a)\right) \\
& \geq d(\gamma(s), B(o, a))+d(\gamma( \pm t), B(o, a))-a \\
& \geq|s|+t+3 a
\end{aligned}
$$

the last inequality follows from the property (I). Clearly, this implies that any minimizing geodesic, say, $\alpha_{t}$, from $p$ to $\gamma^{ \pm}(t)$ does not pass through $B(o, a)$. Hence any convergent subsequence of $\alpha_{t}$ will converge to a ray which is away from $B(o, a)$. This proves the claim.

Next, we claim that through every point of the $a$-tubular neighborhood of $\gamma$, there exists a line with the property (I). Indeed, it follows from the above claim and Lemma 3.1 that through every point of the $a$-tubular neighborhood of $\gamma$, there is a line $\beta$ such that

$$
b_{\gamma}^{+}\left(\beta^{+}(t)\right)=t \text { and } b_{\gamma}^{-}\left(\beta^{-}(t)\right)=t .
$$

We need to show that $\beta$ also has the property (I), i.e.

$$
d(\beta(t), B(o, a)) \geq|t|+2 a \text { for all } t .
$$

By symmetry, we may assume that $t \geq 0$. Then for any $r \geq 0$,

$$
\begin{aligned}
d(\beta(t), B(o, a)) & \geq d(\gamma(r), B(o, a))-d(\beta(t), \gamma(r)) \\
& \geq r-d(\beta(t), \gamma(r))+2 a
\end{aligned}
$$

(here we used the property (I) for $\gamma$ ). Letting $r \rightarrow \infty$ in the above inequality, we have

$$
d(\beta(t), B(o, a)) \geq b_{\gamma}^{+}(\beta(t))+2 a=t+2 a .
$$

Now let $\alpha(t):[0, d] \rightarrow M$ be a minimizing geodesic from $\gamma(0)$ to $o$, then there is a partition of the inteval $[0, d]: t_{0}=0<t_{1}<$ $\cdots<t_{k}=d$ such that $d\left(\alpha\left(t_{i}\right), \alpha\left(t_{i+1}\right)\right)<a$. 
The last claim implies that there is a line through $\alpha\left(t_{1}\right)$ with the property (I). Continuing this process inductively, we would find a line with the property (I) through $\alpha\left(t_{k}\right)$, the base point $o$, which is absurd.

We are now in the position to prove Proposition 2.2.

Proof of Proposition 2.2. Suppose the contrary. That is, $d\left(\gamma_{1}(4 a)\right.$, $\left.\gamma_{2}(4 a)\right) \leq 2 a$. Since $\left[\gamma_{1}\right]$ and $\left[\gamma_{2}\right]$ are different ends, there exists an $A>4 a$ such that $\gamma_{1}(t)$ and $\gamma_{2}(t)$ are in different unbounded components of $M-B(o, A)$ for all $t>A$. Let $C_{t}(t>A)$ be a minimizing geodesic joining $\gamma_{1}(t)$ and $\gamma_{2}(t)$. Then $C_{t}$ must pass through $B(o, A)$. In addition, we claim that the middle point $m_{t}$ of $C_{t}$ is in the ball $B(o, 2 A)$. As a matter of fact, let $p$ be a point in $C_{t} \cap B(o, A)$ and without loss of generality we may assume that $d\left(p, \gamma_{1}(t)\right) \leq d\left(p, \gamma_{2}(t)\right)$, then

$$
\begin{aligned}
d\left(o, m_{t}\right) & \leq d(o, p)+d\left(p, m_{t}\right) \\
& \leq A+\frac{1}{2} \rho_{t}-d\left(p, \gamma_{1}(t)\right) \\
& \leq A+\frac{1}{2} \rho_{t}-(t-A)
\end{aligned}
$$

where $\rho_{t}=$ the length of $C_{t}$. Notice that

$$
\begin{aligned}
\rho_{t} & =d\left(\gamma_{1}(t), \gamma_{2}(t)\right) \\
& \leq d\left(\gamma_{1}(t), \gamma_{1}(4 a)\right)+d\left(\gamma_{1}(4 a), \gamma_{2}(4 a)\right)+d\left(\gamma_{2}(4 a), \gamma_{2}(t)\right) \\
& \leq 2(t-4 a)+2 a=2 t-6 a .
\end{aligned}
$$

Hence,

$$
\begin{aligned}
d\left(o, m_{t}\right) & \leq A+\frac{1}{2}(2 t-6 a)-(t-A) \\
& =2 A-3 a .
\end{aligned}
$$

This shows that $m_{t}$ is in the ball $B(o, 2 A)$.

Now we reparametrize $C_{t}$ by translating the origin and with abuse of notation we still denote it by $C_{t}$ such that

$$
C_{t}\left(-\frac{1}{2} \rho_{t}\right)=\gamma_{1}(t), \quad C_{t}(0)=m_{t}, \quad C_{t}\left(\frac{1}{2} \rho_{t}\right)=\gamma_{2}(t)
$$

We claim that $C_{t}(s)$ satisfies property (I) for $-\frac{1}{2} \rho_{t} \leq s \leq \frac{1}{2} \rho_{t}$. In fact, for any $s$ (we may assume $s \geq 0$ ),

$$
\begin{aligned}
d\left(C_{t}(s), B(o, a)\right) & \geq d\left(C_{t}\left(\frac{1}{2} \rho_{t}\right), B(o, a)\right)-\left(\frac{1}{2} \rho_{t}-s\right) \\
& \geq(t-a)-(t-3 a)+s \\
& =s+2 a
\end{aligned}
$$

where we used the fact $\rho_{t} \leq 2 t-6 a$. Since $C_{t}(0) \in B(o, 2 A)$ for all $t \geq A$, when $t \rightarrow \infty$, a subsequence of $C_{t}$ converges to a line $\gamma(s)$ with the property (I) for all $s$. (Notice that $\rho_{t} \rightarrow \infty$, as $t \rightarrow \infty)$. This is a contradiction by Lemma 3.3. 


\section{REFERENCES}

[A] U. Abresch, Lower curvature bounds, Toponogov's Theorem and bounded topology, Ann. Sci. École Norm. Sup. Paris 28 (1985), 665-670.

[AG] U. Abresch and D. Gromoll, On complete manifolds with nonnegative Ricci curvature, J. Amer. Math. Soc. (to appear).

[CG] J. Cheeger and D. Gromoll, The Splitting Theorem for manifolds of nonnegative Ricci curvature, J. Differential Geom. 6 (1971), 119-128.

[EH] J. Eschenburg and E. Heintze, An elementary proof of the Cheeger-Gromoll Splitting Theorem, Ann. Global Anal. Geom. 2 (1984), 141-151.

[L] Z. Liu., Ball covering on manifolds with nonnegative Ricci curvature near infinity, SUNY at Stony Brook, preprint, 1990.

[LT] P. Li and L. F. Tam, Harmonic functions and the structure of complete manifolds, University of Arizona, preprint, 1990.

[T] V. A. Toponogov, Riemannian spaces which contain straight lines, Amer. Math. Soc. Transl. (2) 37 (1964), 287-290.

Department of Mathematics, University of Pennsylvania, PhiladelPHiA, PenNSYlVANia 19104

E-mail address: zhang@grasp.cis.upenn.edu 
